\title{
Les manifestations scientifiques et techniques organisées par la SFRP
}

\author{
D. BLANC ${ }^{1}$, P. BALLEREAU ${ }^{1}$
}

\begin{abstract}
RÉSUMÉ Le devoir premier d'une société comme la SFRP est l'organisation de congrès, colloques, journées portant sur des sujets jugés susceptibles d'intéresser ses sociétaires. Cela implique l'existence d'une structure spécialisée, et compétente. Jusqu'en 1992, ce fut la commission des programmes scientifiques et techniques, devenue progressivement tyrannique. En 1992, la Société fit un grand effort de décentralisation, et remplaça cette structure rigide par un certain nombre de sections, indépendantes les unes des autres. Cette nouvelle organisation eut un succès immédiat ; elle a maintenant fait ses preuves, grâce à sa souplesse, mais aussi à son efficacité.
\end{abstract}

ABSTRACT Scientifics and technical meeting organised by SFRP.

The first objective of the SFRP is to organize congress and workshops on topics interesting its members. To organise such meeting a competent staff is necessary. Until 1992 it was the job of a specific commission inside the society but its role became too much tyrannical. In 1992, the society transferred its role to independent technical sections. The success was immediately complete and today it is always the way used for each workshop.

\section{Avant-propos}

Un article va être consacré à l'évolution de notre revue depuis sa création. Si la revue est un élément indispensable des activités de la SFRP, il faut souligner que le rôle premier d'une société dite « savante » est l'organisation de manifestations relevant de sa spécialité. Ces manifestations assurent son prestige, mais contribuent aussi à couvrir ses frais de fonctionnement.

Durant les cinq premières années de son existence, la SFRP créa trois commissions : juridique et du règlement, expansion, publications. Rien ne concernait, spécifiquement, les réunions, journées, colloques, congrès, si ce n'est un comité, dirigé par Francis Duhamel, mais non entériné par l'assemblée générale (AG). Le 5 juin 1970, le conseil officialise cette situation : il crée une commission chargée du choix et de l'organisation des manifestations, et en confie la présidence à Francis Duhamel.

SFRP, BP 72, 92263 Fontenay-aux-Roses, France. 
On trouvera sur le site de la SFRP (www.sfrp.asso.fr) la liste complète des manifestations organisées par la Société depuis sa création. Cet article fait le point sur la période 1970-1990 (Ballereau, 1990).

\section{De 1970 à 1992 : la commission des programmes scientifiques et techniques}

Cette commission, vers la fin de son parcours, a changé de nom, mais son esprit est resté le même.

\subsection{Création de la commission}

Francis Duhamel préside la SFRP en 1969-1970. À l'issue de sa présidence (un an, à cette époque), il rebondit en prenant la présidence de la nouvelle commission. Dans son rapport moral devant l'AG du 5 juin 1970, il écrit: « Le conseil d'administration m'a personnellement demandé de prévoir et de mettre sur pied une quatrième commission, celle des activités de la société. Il s'agirait essentiellement de prévoir les réunions ordinaires, et de préparer les mesures propres à favoriser les échanges entre les adhérents »(Duhamel, 1970).

Dans son rapport moral, lors de l'AG du 4 juin 1971, le président sortant, Maurice Avarguès écrit: "Le conseil d'administration avait, dès la première réunion du nouvel exercice, marqué sa volonté de développer un effort tout particulier dans le domaine de la tenue de nos réunions scientifiques et techniques. Ce fut la tâche principale du comité des réunions scientifiques et techniques, animé par M. Duhamel, notre président sortant, et par l'équipe active et dynamique dont il est entouré » (Avarguès, 1971).

Francis Duhamel est entouré de MM. Brodsky, Michel Diéval, Lucien Fitoussi et Georges Marblé [1], nommés par le conseil à compter du $1^{\text {er }}$ octobre 1970. Francis Duhamel présidera ce comité, puis commission, durant onze années, les effectifs grossissant progressivement, ainsi que le prévoit la loi d'évolution des groupes : on en est à 23 membres en 1980.

Paradoxalement, ce n'est qu'en 1978 que l'AG est appelée à approuver l'existence de la commission et son appellation «commission des programmes scientifiques et techniques » ou CPST : on avait oublié de la consulter (Marchand, 1978). Nous y reviendrons.

\subsection{L'acteur principal, Francis Duhamel}

Comme on le sait (Blanc, 2003), la SFRP est, pour faire simple, la synthèse de deux groupes, les « ingénieurs », Francis Duhamel, et les «médecins », Henri 
Jammet. Il y a une compétition, souterraine, de prépondérance et de pouvoir entre les deux groupes. Durant sa présidence, Francis Duhamel avait préparé la pérennité du comité qu'il préside.

Francis Duhamel fait grossir progressivement le volume de sa commission. Le 5 juin 1970, avant même qu'elle ne se soit réunie, il propose au conseil de subdiviser la commission en un certain nombre de comités. Agacé, le président Maurice Avarguès " propose que l'on commence par assurer le fonctionnement de cette commission pour les activités scientifiques (réunions) avant d'envisager de l'étendre à d'autres activités » [2].

Durant plus de neuf années, la commission fait son travail avec sérieux et compétence. Mais, à l'issue de cette période, le remplacement de son président qui, en fait, est présent depuis 14 ans - va être problématique.

\subsection{Un passage difficile (1979-1980), la succession de Francis Duhamel}

Le conseil fait un appel à candidatures ; leur examen est inscrit à l'ordre du jour de la réunion du 21 décembre 1979 [3]. Lucien Fitoussi dépose sa candidature, mais la retire la veille de la réunion du conseil. Restent en lice deux candidats, Pierre Bovard et Pierre Jockey. Le premier est ingénieur, le second est médecin. Vous avez compris ? Eh bien, c'est l'inverse : Pierre Jockey est un ami de Francis Duhamel, Pierre Bovard est plutôt neutre.

La profession de foi de Pierre Bovard est présentée par le secrétaire général de la SFRP, qui lit la lettre du candidat au président. En voici un passage : «Pour mon programme, je propose la continuation de l'auvre entreprise par $M$. Duhamel et la répartition des tâches entre les membres les plus actifs de la commission. Pour ma part, je désirerais orienter, si j'obtenais votre confiance, mes projets vers les problèmes posés par la protection du personnel de l'industrie nucléaire, et soulevés par l'opinion publique, avec pour objectif de ne pas sacrifier la protection du personnel à une protection inconsidérée de l'environnement, d'une part, et, d'autre part, mieux informer le grand public des impacts réels et non imaginaires ou passionnels de l'énergie nucléaire. »

Pierre Jockey est membre du conseil. Il prend la parole et propose les orientations suivantes: "Développer les réunions à audience internationale, multiplier les fonctions d'enseignement, appuyer les groupes techniques mis en place ces derniers mois, assurer la publication rapide des actes de toutes les réunions scientifiques. » 
On passe au vote. $1^{\text {er }}$ tour de scrutin : Bovard, 7 voix ; Jockey, 7 voix. $2^{\mathrm{e}}$ tour de scrutin : Bovard, 7 voix ; Jockey, 7 voix. C'est l'impasse. Le conseil propose à Francis Duhamel, qui accepte, de se succéder à lui-même et d'assurer l'intérim de la présidence de la CPST jusqu'à la réunion du conseil qui suivra l'AG de 1980.

L'élection du successeur doit donc avoir lieu en octobre 1980. Le 19 septembre [4], afin d'y voir plus clair, le conseil cherche à redéfinir le rôle de la commission. Le président Raymond Bardoux rend hommage à Francis Duhamel, qui a fortement développé les activités de la commission, qui «arrivent à l'occuper presque à temps complet ». C'est trop, et le conseil pense qu'il serait bon de créer des «comités » ou des « groupes » indépendants de la commission, par exemple rayonnements non ionisants, formation, afin d'alléger les tâches, mais Francis Duhamel s'oppose fermement à tout ce qui peut diminuer l'influence de la commission. Il propose : «la commission pourrait comporter, soit un président, soit un délégué du président de la SFRP, qui réunirait les présidents des comités, et les ferait travailler ensemble. » La discussion s'éternise, chacun restant sur ses positions. Afin de clore le débat, le président met aux voix la proposition suivante :

"LA SFRP comprendra : trois commissions (revue, juridique et du règlement, réunions scientifiques et techniques) et deux groupes techniques indépendants (rayonnements non ionisants, formation). "

Le scrutin donne 5 voix pour cette proposition, 4 voix contre (maintien $\mathrm{du}$ statu quo), 4 abstentions. La situation est donc maintenue en l'état; cette discussion a été inutile, apparemment tout au moins.

Le 29 octobre 1980, le conseil doit élire le nouveau président de la CPST [5]. Francis Duhamel, président par intérim, continue à défendre une CPST puissante, ne déléguant que chichement - et avec un post-contrôle - ses pouvoirs à des "groupes », «comités », voire «sections ». Francis Duhamel annonce que les membres de la CPST proposent, à l'unanimité, la candidature de Pierre Jockey.

Or, lors de sa dernière réunion, le bureau a proposé que «l'on confie au président élu, M. Coulon, et ce jusqu'au conseil d'administration suivant la prochaine assemblée générale, l'intérim de la présidence de la CPST, en vue de procéder à une réflexion sur une éventuelle restructuration de cette commission". Le président met aux voix cette proposition, à bulletins secrets ; elle est repoussée par 8 voix contre 7 .

Rien n'avançant, afin de déverrouiller, le conseil procède à un tour de scrutin purement indicatif, afin de dégager des candidatures au poste de président de la CPST. Le résultat est le suivant: Philippe Beau, 2 voix ; René Coulon, 4 voix ; Pierre Jockey, 5 voix; 4 bulletins blancs. Seul Pierre Jockey maintient sa 
candidature. On vote sur son nom : oui, 8 ; non, 5 ; un vote blanc, un vote nul. Pierre Jockey est élu président de la CPST.

\subsection{La présidence de Pierre Jockey}

Tel un premier ministre, il forme son gouvernement (pardon, sa commission), soit 27 personnes (Tab. I). Le conseil l'approuve le 5 janvier 1981 [6]. Malgré son obésité, la commission va encore vivre une dizaine d'années, bien qu'elle perde peu à peu son influence. On remarquera que le groupe «rayonnements non ionisants » est incorporé dans la commission, sur le papier, tout au moins.

Pierre Jockey termine son mandat le 23 mars 1983 au bout d'un peu plus de deux ans. Ont été organisées neuf journées, deux séminaires, un congrès international en coopération avec la société italienne de radioprotection.

\section{TABLEAU I}

Composition de la commission des programmes scientifiques et techniques de la SFRP, au $1^{\text {er }}$ janvier 1981.

Members of the scientific and technical programs commission, SFRP, January 1st, 1981.

P. Jockey, président
F. Duhamel, coordination des groupes de travail, responsable du groupe enseignement et formation. Adjoint
J. Cluchet

A.J. Berteaud (suppléante, $M^{\text {me }}$ A. Duchène), groupe rayonnements non ionisants

Bastin (suppléant, J.-M. Chinardet), groupe information technique

P. Candès (suppléante, $M^{\mathrm{me}}$ A. Carnino), groupe sûreté

Rouhet (suppléant, J.Marquant), ergonomie

H. Frossard, questions médicales

J. Sarbach, questions médicales

$\mathrm{M}^{\mathrm{me}} \mathrm{S}$. Vignes, liaison avec EDF et SFEN

$\mathrm{M}^{\mathrm{mc}} \mathrm{M}$. Archimbaud (suppléant, $\mathrm{P}$. Marchand), liaison avec le groupe Sud-Est, questions d hygiène industrielle

P. Bovard, liaison avec l'ANDRA, questions concernant les déchets

J.-M. Chinardet, biologie el médecine

R. Choquet (suppléant, J. Frank), liaison avec les universités

J. Cluchet, liaison avec l'ATSR et les SPR

C. Chastel (suppléant, C. Berthier), liaison avec la sécuritê civile

R. Pachet, liaison avec l'AFTIM et les ministères, problèmes de sécurité

B. Werderer, sécurité et chimie

L. Fitoussi, réunions avec les sociétés étrangères

G. Bardet, problèmes de déchets,

P. Ballereau, assistant du président pour les questions d'organisation, de planning. de secrétariar

$\mathrm{M}^{\mathrm{mc}}$ Altmeyer, secrétaire de la commission

R. Coulon, président élu de la SFRP, es qualité 


\subsection{Les présidents suivants}

Jean Scheidhauer est élu par le conseil le 4 mai 1983. Il démissionne au $1^{\text {er }}$ octobre 1986, et propose que Jean Cluchet lui succède. La passation des « pouvoirs » a lieu le 8 octobre 1986 [7], lors de la réunion sur le transport des matières radioactives. $\mathrm{Au}$ cours de son mandat, la commission organise 17 réunions, dont 10 du type colloque, ainsi que trois réunions internationales (deux avec la société italienne, une avec la société belge).

Jean Cluchet achève son mandat en avril 1989, car il est gravement malade, et décède en avril 1990. Le 10 octobre 1989 [8], le conseil confie la présidence, à titre provisoire, à Lucien Fitoussi ; il conservera ce mandat après le décès de Jean Cluchet. Il termine ses fonctions le 3 juin 1992, en présidant la quatre-vingt-dixseptième, et dernière, réunion de la commission.

En effet, rien ne va plus, car ont été créées des sections scientifiques ou techniques qui, indépendantes, effondrent rapidement le pouvoir et les activités de la CPST.

\subsection{Disparition de la commission}

Le 25 février 1992, le conseil [9] transforme la CPST en « commission de coordination scientifique et technique », ou CCST, et lui enlève une grande partie de ses attributions. Lucien Fitoussi, qui assiste à la réunion, «estime que les sections doivent transmettre leurs projets de réunions à la future CCST, qui les discutera pour les entériner dans le cadre des directives du conseil d'administration ». Pour sa part, Mme Monique Vuillaume propose que "les sections transmettent leur programme d'abord au conseil d'administration, qui donne ou non son accord, et demande - éventuellement - l'avis de la CCST».

Le président Laurent Stricker s'exprime avec beaucoup de clarté [9] : «Les sections doivent avoir la responsabilité de prendre les initiatives qu'elles jugent utiles. Elles constitueront l'âme de la société, et lui permettront de s'épanouir autour de thèmes structurants. "

\section{Depuis 1992 : les sections scientifiques et techniques}

\subsection{Les prémices}

On trouve la clef de l'évolution de la SFRP dans les décisions prises par l'AG extraordinaire du 22 juin 1978, faisant suite à l'AG statutaire (Marchand, 1978). Cette AG s'est déroulée sans passion ; probablement involontairement, elle oriente la SFRP vers une autre manière de travailler. Donnons quelques précisions. 
Depuis 1970, la commission de Francis Duhamel est dans l'illégalité, car non approuvée par l'AG. Le président Régis Marchand salue «la création d'une commission désormais réglementaire des programmes scientifiques et techniques, dont la mission serait de proposer au conseil les programmes d'activité correspondant à la vocation de la Société et notamment de coordonner les activités scientifiques menées éventuellement au sein des groupes régionaux ou de groupes techniques créés par le conseil d'administration pour connaitre de questions particulières si cela paraît nécessaire ».

Il est question de créer, parallèlement à la CPST, une commission « des actions scientifiques et techniques », restée fumeuse, car devant se trouver, inévitablement, en conflit avec la CPST. Cette commission ne sera jamais créée.

Mais écoutons le président Régis Marchand : «Je me permets d'insister sur l'importance des en têtes spécialisés dont l'existence est prévue au sein de l'éventuelle commission des actions scientifiques et techniques, sous forme de groupes techniques car j'y vois l'amorce de structures d'accueil où pourront se retrouver ceux qui s'intéressent aux formes nouvelles de la radioprotection, concernant par exemple les micro-ondes. »

D'un côté, l'AG donne sa bénédiction à la CPST. De l'autre, elle introduit le concept de groupes techniques qui concernent, certes, des sujets nouveaux, mais qui, à terme, seront conduits à traiter de sujets plus classiques.

La création de groupes dynamiques et indépendants, travaillant sur des domaines de pointe va briser le carcan mis en place par la CPST. Cette commission perd lentement son influence quand de tels groupes atteignent des tailles suffisantes. Mais tout cela va demander plus de dix ans.

\subsection{La création des sections}

Le premier groupe apparaît en 1979, à la suite de l'AG que nous venons de décrire. C'est le « groupe de travail sur les rayonnements non ionisants ", très jaloux de son indépendance. Il est animé par André-Jean Berteaud, maître de recherche au CNRS. Le conseil officialise ce groupe en 1981, avec pour responsables AndréJean Berteaud et Annette Duchène (CEA). Le tableau I le montre intégré dans la CPST, mais c'est uniquement sur le papier.

D'autres groupes sont en gestation. Il s'agit de « l'information technique en matière de radioprotection », et de « l'ergonomie dans les utilisations de l'énergie nucléaire ». Aucun ne verra le jour officiellement. 
En 1988, le conseil lance l'idée d'une association avec l'ATSR (association des techniciens supérieurs en radioprotection, devenue association des techniciens et scientifiques en radioprotection), à travers une section technique. Le président Jacques Pradel souligne que, même sans l'appui de l'ATSR, une telle section devra être créée. C'est bien ce qui se produit. Le 2 mai 1990 [10], le conseil crée la section de « protection technique » ; l'AG du 18 juin 1990 confirme cette création. La section est présidée par Solange Descours. Le 10 octobre 1990, le conseil [11] modifie certains aspects de la mission de cette section, mais il stipule que cette mission comporte, notamment, l'organisation de journées de travail sur invitations, des réunions élargies organisées en coopération avec la CPST, des visites.

Sous l'impulsion de Monique Vuillaume (anonyme, 1991), l'AG de juin 1991 crée la section « recherche et santé ».

Quant au groupe « Sud-Est » de la SFRP, seul groupe régional de la SFRP, très dynamique, il est métamorphosé en 1992 [9] en section « Environnement », pour des raisons assez complexes d'opportunité.

Le 16 décembre 1991, le conseil souligne fortement l'existence de quatre sections: protection technique, recherche et santé, environnement et rayonnements non ionisants, aboutissements du groupe de travail créé en 1979. Ces sections travaillent indépendamment de la CPST qui est « chargée de la coordination des manifestations scientifiques et du contrôle de la cohérence de l'action des sections », c'est-à-dire qu'elle s'efface.

Cette mutation sans effusion de sang est l'œuvre du président Laurent Stricker. Voici, en guise de conclusion, des extraits de ses écrits :

Lors de l'AG du 30 juin 1992 (Stricker, 1992), il souligne l'importance des sections : «Ces dernières, lancées il y a trois ans, ont pris leur essor, et devraient créer un dynamisme sur lequel il va falloir s'appuyer ».

Dans son rapport moral (Stricker, 1992), il écrit : "L'évolution de la SFRP tourne autour du fonctionnement des sections techniques. Celles-ci sont un peu le fonds de commerce de la SFRP, dont elles font vivre les différentes forces, en organisant des réunions bien ciblées sur les centres d'intérêt des uns et des autres. »

Dans son rapport moral du 29 juin 1993 (Stricker, 1993), il ajoute : « Le champ d'action de la SFRP est considérablement élargi sous l'impulsion des sections, dont je tiens à souligner ici l'importante action, qui est à la base de la qualité du travail effectué par la société. » 


\section{Note importante}

Documents non publiés, cités dans le texte.

[1] Compte rendu d'activité du comité des programmes scientifiques et techniques, 6 octobre 1971.

[2] Document 70/2007, PV (procès verbal) du CA (conseil d'administration) du 5 juin 1970.

[3] Document 79/106, PV du CA du 21 décembre 1979.

[4] Document 80/046, PV du CA du 19 septembre 1980.

[5] Document 80/055, PV du CA du 29 octobre 1980.

[6] Document 81/007, PV du CA du 5 janvier 1981.

[7] PV 99/86, CA du 2 octobre 1986.

[8] PV 112 88/89, CA du 10 octobre 1989.

[9] PV 122 91/92, CA du 2 mai 1990.

[10] PV 114 89/90, CA du 2 mai 1990.

[11] PV 115 89/90, CA du lo octobre 1990.

\section{RÉFÉRENCES}

Anonyme (1991) Création d'une section « recherche et santé », Radioprotection 26, 705-706.

Avarguès M. (1971) Rapport moral, Radioprotection 6, 221-222.

Ballereau P. (1990) Historique de la commission des programmes scientifiques et techniques, 1970 1990. Document distribué aux membres du conseil et aux présidents des commissions. Archives de la SFRP.

Blanc D. (2003) La naissance de la SFRP, Radioprotection, 38, 217-223.

Duhamel F. (1970) Rapport moral, Radioprotection 5, 251-255.

Marchand M. (1978) Rapport moral, Radioprotection 13, 199-203.

Stricker L. (1992) Rapport moral, Radioprotection 27, 344-345.

Stricker L. (1993) Rapport moral, Radioprotection 28, 349-350. 